\title{
Blunt traumatic aortic injuries of the ascending aorta and aortic arch: a clinical multicentre study
}

\author{
Victor X. Mosquera, Milagros Marini, Javier Muñiz, Daniel Gulias, Vanesa Asorey- \\ Veiga, Belen Adrio-Nazar, José M. Herrera, Gonzalo Pradas-Montilla, José J. Cuenca
}

\begin{abstract}
Objective. To report the clinical and radiological characteristics, management and outcomes of traumatic ascending aorta and aortic arch injuries.

Methods. Historic cohort multicentre study including 17 major trauma patients with traumatic aortic injury from January 2000 to January 2011.

Results. The most common mechanism of blunt trauma was motor-vehicle crash (47\%) followed by motorcycle crash (41\%). Patients sustaining traumatic ascending aorta or aortic arch injuries presented a high proportion of myocardial contusion $(41 \%)$; moderate or greater aortic valve regurgitation $(12 \%)$; haemopericardium (35\%); severe head injuries (65\%) and spinal cord injury (23\%). The 58.8\% of the patients presented a high degree aortic injury (types III and IV). Expected in-hospital mortality was over $50 \%$ as defined by mean TRISS 59.7 (SD 38.6) and mean ISS 48.2 (SD 21.6) on admission. Observed in-hospital mortality was 53\%. The cause of death was directly related to the ATAI in $45 \%$ of cases, head and abdominal injuries being the cause of death in the remaining $55 \%$ cases. Long-term survival was $46 \%$ at 1 year, $39 \%$ at 5 years, and $19 \%$ at 10 years.

Conclusions. Traumatic aortic injuries of the ascending aorta/arch should be considered in any major thoracic trauma patient presenting cardiac tamponade, aortic valve regurgitation and/or myocardial contusion. These aortic injuries are also associated with a high incidence of neurological injuries, which can be just as lethal as the aortic injury, so treatment priorities should be modulated on an individual basis.
\end{abstract}

\section{Keywords}

Aorta; Aortic arch; Trauma surgery; Emergency

\section{Introduction}

The aortic isthmus is by far the most common site of acute traumatic aortic injury (ATAI), but up to $10 \%$ of cases of ATAI occur in atypical locations including the aortic arch, the ascending aorta and the peridiaphragmatic aorta. 1,2 and 3 Among those atypical sites of ATAI, the ascending aorta and aortic arch injuries outstand because of their ominous prognosis. They are highly lethal as most victims die at the scene of the accident or during transportation. ${ }^{4}$ and 5 Although improvement in restraints has decreased the incidence of ascending aorta and aortic arch traumatic injuries related to motor-vehicle collisions in last decades, ${ }^{1 \text { and } 6}$ the prognosis of those aortic injuries remains poor. The management of ATAI of ascending aorta and aortic arch and its effect on outcomes are less documented than those of injuries at the isthmus and beyond. Most studies in the last decade about management of ATAI have only focused on injuries at the level of the aortic isthmus and descending aorta, which are more amenable to thoracic endovascular aortic repair (TEVAR), there being a lack of up-to-date information about the management and long-term outcomes of ATAI of the ascending aorta and aortic arch.

The objective of this study is to report the clinical and radiological characteristics, management and early and long-term survival of a multicentre series of traumatic ascending aorta and aortic arch injuries. 


\section{Patients and methods}

This is a historic cohort multicentre study with 3 participating institutions, which are the reference firstlevel trauma centres for ATAI in a Spanish region of more than 2,700,000 inhabitants. A total of 85 consecutive major trauma patients with ATAI were admitted from January 2000 to January 2011, among which 17 major trauma patients with traumatic injury at the ascending aorta or the aortic arch were identified. No patient who reached alive to hospital sustained traumatic aortic injuries at multiple locations.

17 patients with ATAI at ascending aorta or the aortic arch between 2000-2010

HOSPITAL

DISCHARGE

END FOLLOW-UP

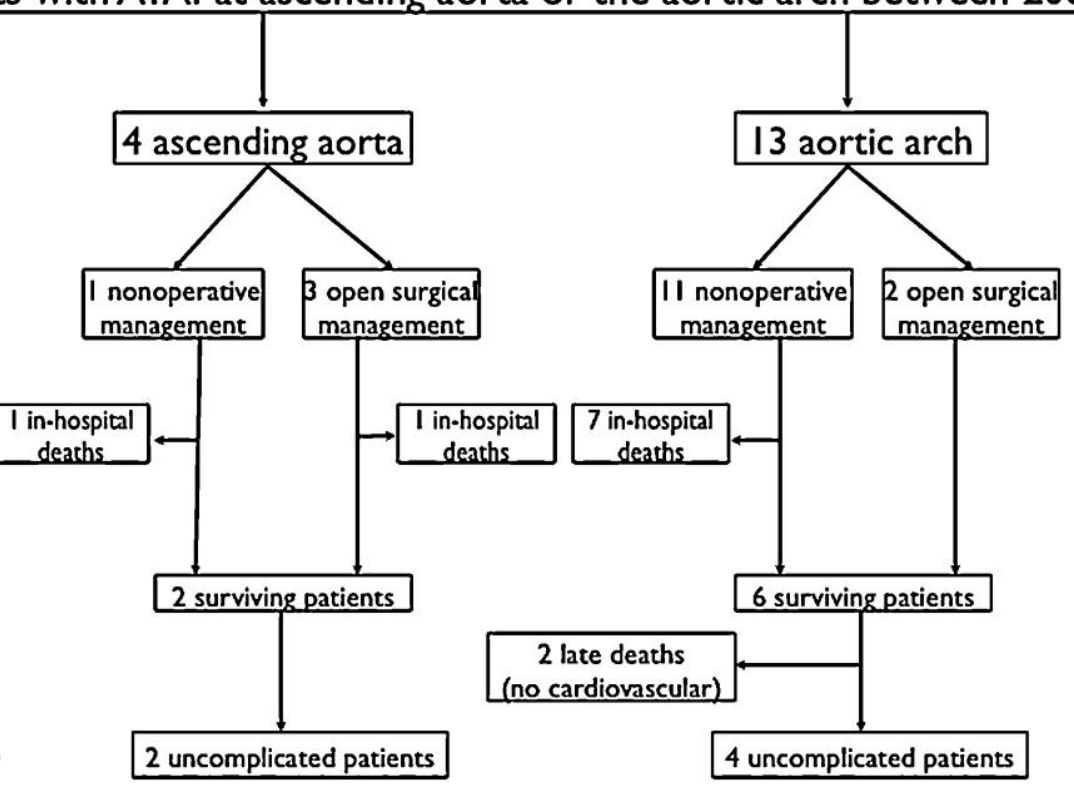

Fig. 1 depicts a flow diagram describing the design of the study and the flow of patients.

Data on 96 variables were recorded on a standardised form that included information on patient demographics, mechanism of injury, initial clinical presentation (blood pressure, respiratory rate, need of endotracheal intubation at the site of the trauma or during transport, Glasgow Coma Scale [GCS]), Injury Severity Score (ISS), ${ }^{7}$ Abbreviated Injury Score (AIS) for each body area (head, chest, abdomen, extremities), Revised Trauma Score (RTS), ${ }^{8}$ Trauma Injury Severity Score (TRISS), ${ }^{9}$ Traumatic Aortic Injury Score (TRAINS), ${ }^{10}$ head and neck injuries, nonmediastinal thoracic injuries, cardiac injuries, abdominal injuries, pelvic fracture, extremities fractures, findings on admission simple chest X-ray, performed diagnostic imaging tests (computed tomography (CT), angiography, transthoracic and/or transoesophageal echocardiogram (TEE)).

An ISS score of more than 50 points predicts a mortality rate of over $50 \%$, while a score of more than 70 points predicts a mortality rate of nearly $100 \% .^{7}$ The TRISS score directly predicts the expected death rate for blunt trauma. ${ }^{9}$

A TRAINS score equal or greater than 4 points is highly predictive of the probability of presenting an ATAI in major trauma patients. ${ }^{10}$

The aortic injuries were classified according to their severity in type I (intimal tear), type II (intramural haematoma), type III (pseudoaneurysm), or type IV (rupture) following clinical practice guidelines of the Society for Vascular Surgery. ${ }^{11}$ The type of definitive management (conservative treatment or surgical repair) was also recorded.

The mechanisms of blunt trauma were classified as: motor-vehicle crash; motorcycle crash; fall; pedestrian-vehicle accident; crush under weight, and others. Hypotension was defined as a systolic blood pressure $<90 \mathrm{mmHg}$ or the need of fluid and/or inotropic support to maintain a blood pressure $\geq 90 \mathrm{mmHg}$. An abnormal respiratory rate was defined as bradypnea $<10 \mathrm{breaths} / \mathrm{min}$ or tachypnea $>30$ breaths/min. A GCS below 9 points was defined as cut-off value for neurological bad prognosis on 
admission. Head injury was defined as skull fracture, unconsciousness at evaluation, subarachnoid haemorrhage, epidural or subdural haemorrhage.

In cases of motor-vehicle crash patients, information on vehicular speed and seat belt and/or other restraint systems use was not generally available and was not studied.

Diagnosis was based on imaging (TEE, angiography and/or CT scan) and, when available, confirmation was provided by surgical visualisation and/or autopsy. There was no disagreement in data provided by imaging studies. All patients who presented an ATAI at the ascending aorta or the aortic arch underwent a TEE evaluation of the aortic valve and the severity of an acute aortic regurgitation (AR), when present, was graded on the basis of published criteria in mild, moderate or severe AR. ${ }^{12}$ An AR greater than moderate was considered significant. ${ }^{12}$ All participating centres used the same CT scan acquisition protocols for trauma patients requiring advanced imaging tests.

Penetrating trauma was exclusion criterion in the study. All participating centres used the same the inclusion/exclusion criteria.

The Institutional Review Board approved this study based on retrospective data retrieval, waiving for individual consent.

\section{Conservative treatment}

Medical treatment consisted of strict control of both contractility and blood pressure by continuous intravenous infusion of a vasodilator, and limitation of intravenous fluid infusion once the systolic blood pressure exceeded $100 \mathrm{mmHg}$. Initial medical stabilisation with beta-blockers controlled these parameters reducing heart rate and blood pressure to the lowest amounts that still maintain adequate end-organ perfusion. The systolic blood pressure was titrated to approximately $100 \mathrm{mmHg}$ and the heart rate to $<60 \mathrm{bpm}$. Unless there were contraindications, labetalol was our drug of choice. Anti-hypertensive therapy was changed to oral administration once the patient was haemodynamically stable enough. This practice only deviated in the respect that patients with evidence of increased intracranial pressure were considered immediate operative candidates in order to prevent secondary brain injury associated with the decrease in cerebral perfusion pressure that accompanied hypotensive medical therapy. ${ }^{1}$

Regular radiological follow-up was indicated after discharge with a control thoracic CT scan at 1, 3, 6 and 12 months and then annual cardiovascular magnetic resonance.

\section{Surgical repair}

Cardiopulmonary bypass (CPB) was established by peripheral cannulation of the right axillary or the femoral artery and placement of a single right atrial venous cannula. The injured aorta was approached through a median sternotomy. In cases of aortic arch transection in proximity to the innominate or left common carotid, anterior exposure via extended sternotomy or thoracosternotomy offered better exposure for total arch replacement. Core temperature was allowed to drift to $32{ }^{\circ} \mathrm{C}$, except for cases requiring deep hypothermic circulatory arrest (DHCA). In cases of aortic arch transection in proximity to the innominate or left common carotid, the patient was cooled to a nasopharyngeal temperature below $18{ }^{\circ} \mathrm{C}$. When DHCA was required, antegrade cerebral perfusion (ACP) was performed either through a right axillary artery cannulation or through selective cannulation of the innominate and left carotid arteries with blood between $6^{\circ} \mathrm{C}$ and $12{ }^{\circ} \mathrm{C}$ (flow $10-15 \mathrm{~mL} / \mathrm{kg} / \mathrm{min}$ ).

On the basis of the time from injury to definitive aortic repair, an emergent repair was defined as that performed in the first $24 \mathrm{~h}$ after hospital admission, while a delayed repair was accomplished beyond the first $24 \mathrm{~h}$ after hospital admission.

No patient with ATAI at the ascending aorta or the arch underwent TEVAR.

\section{Statistical analysis}

Data are expressed as mean and standard deviation or median and range, when appropriate. For bivariate analysis, proportions were compared with contingency tables by means of chi-square with Yates' correction or Fisher's exact tests when appropriate, while Student's $t$ test was used to compare continuous variables. A $p$ value of less than 0.05 was considered significant. ${ }^{13}$

Actuarial estimates of survival were accomplished with Kaplan-Meier methods.

The study adheres to the STrengthening the Reporting of OBservational studies in Epidemiology (STROBE) initiative. ${ }^{14}$

The SPSS statistical program for Windows version 17.0 (SPSS, Chicago, IL) was used to perform data analysis. 


\section{Results}

The clinical and demographic characteristics of patients are summarised in Table 1.

Table 1. Epidemiological and clinical characteristics in trauma patients with traumatic injury of ascending aorta or aortic arch (TRAINS = Traumatic Aortic Injury Score; ISS = Injury Severity Score; AIS = Abbreviated Injury Score; RTS $=$ Revised Trauma Score; TRISS = Trauma Injury Severity Score; $\mathrm{SAH}=$ subarachnoid haemorrhage; $\mathrm{SDH}=$ subdural haemorrhage).

\begin{tabular}{|c|c|}
\hline Variables & Patients $(n, \%)(N=17)$ \\
\hline Male & $15(88)$ \\
\hline Age (mean (SD)) & $48.5(20.2)$ \\
\hline Age $>55$ years & $7(41)$ \\
\hline TRAINS $\geq 4$ points & $17(100)$ \\
\hline ISS (mean (SD)) & $48.2(22)$ \\
\hline RTS (mean (SD)) & $5.5(1.9)$ \\
\hline TRISS (mean (SD)) & $59.7(38.6)$ \\
\hline \multicolumn{2}{|l|}{ Mechanism of injury } \\
\hline Motor vehicle crash & $8(47)$ \\
\hline Motorcycle crash & $7(41)$ \\
\hline Fall & 0 \\
\hline Pedestrian-vehicle accident & 0 \\
\hline Crush under weight & $1(6)$ \\
\hline Others & $1(6)$ \\
\hline \multicolumn{2}{|l|}{ Thoracic injuries } \\
\hline Lung contusion & $15(88)$ \\
\hline Haemothorax & $14(82)$ \\
\hline Diaphragm rupture & 0 \\
\hline Myocardial contusion & $7(41)$ \\
\hline Haemopericardium & $6(35)$ \\
\hline Aortic valve regurgitation & $2(12)$ \\
\hline Sternal fracture & $3(18)$ \\
\hline Rib fracture & $11(65)$ \\
\hline Clavicle fracture & $3(18)$ \\
\hline Scapula fracture & $2(12)$ \\
\hline \multicolumn{2}{|l|}{ Abdominal injuries } \\
\hline Spleen & $1(6)$ \\
\hline Liver & $3(18)$ \\
\hline Kidney & $1(6)$ \\
\hline Bowel & $2(12)$ \\
\hline Bladder & 0 \\
\hline Haemoperitoneum & $5(29)$ \\
\hline \multicolumn{2}{|l|}{ Pelvic and extremity injuries } \\
\hline Pelvis & $7(41)$ \\
\hline Long bone fracture (upper/lower) & $9(53)$ \\
\hline Spinal fracture & $5(29)$ \\
\hline \multicolumn{2}{|l|}{ Head and neck injury } \\
\hline Spinal cord injury & $4(23)$ \\
\hline $\mathrm{SAH} / \mathrm{SDH}$ & $9(53)$ \\
\hline
\end{tabular}

Fig. 2 shows imaging tests in major trauma patients confirming the presence of injuries at the ascending aorta or the arch. 


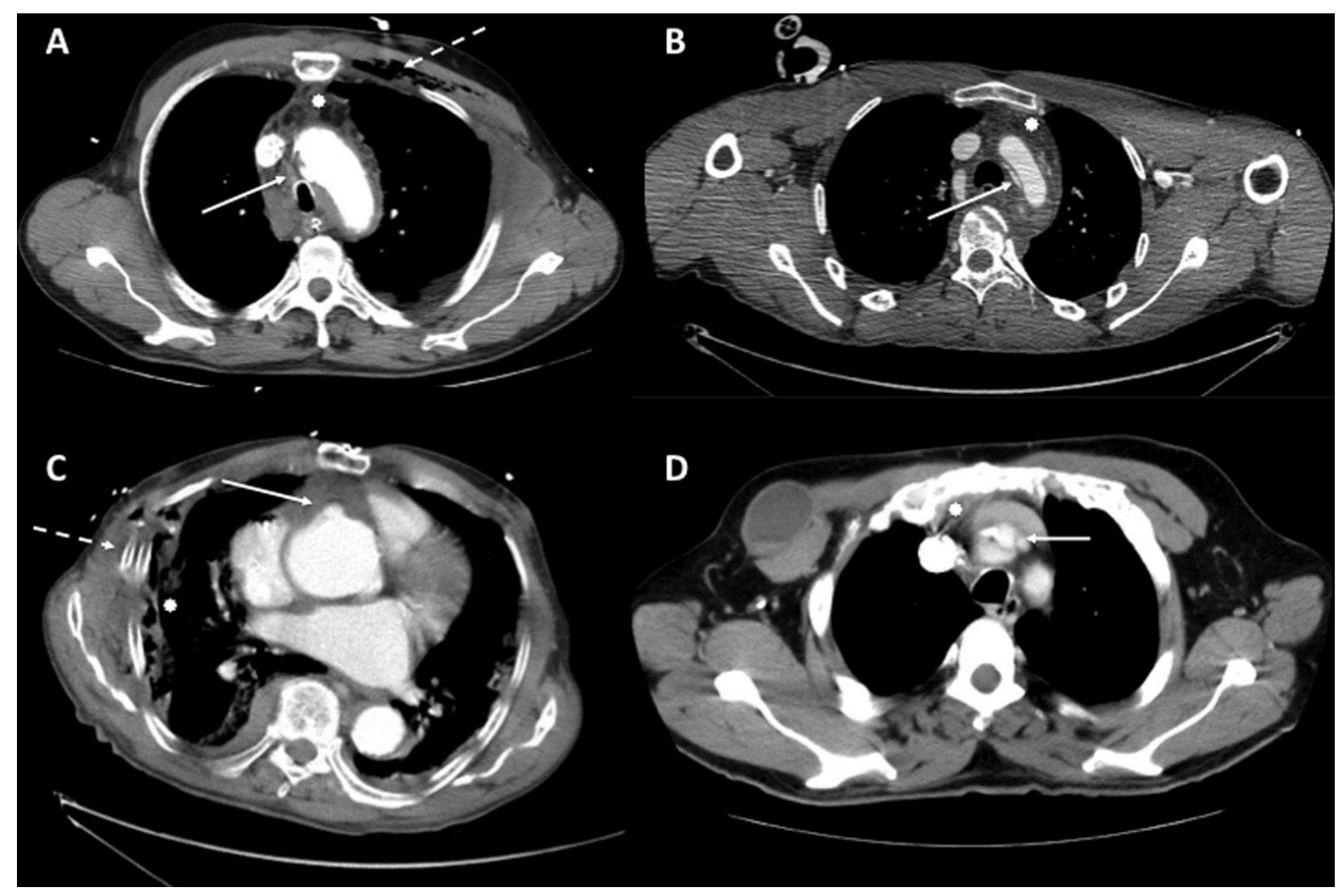

Fig. 2. (A) Thoracic MDCT axial slide shows a traumatic aortic pseudoaneurysm at the inner curve of the aortic arch (solid arrow) with anterior mediastinal haematoma (asterisk). Notice multiple fractures of the left upper chest wall (dashed arrow). (B) Thoracic MDCT axial slide shows a traumatic transection of the aortic arch (solid arrow) with anterior mediastinal haematoma (asterisk). (C) Thoracic MDCT axial slide demonstrates a traumatic injury of the aortic root with an image of "outpouching" (solid arrow). Notice the severe right lung contusion (asterisk) and multiple rib fractures of the right upper chest wall with subcutaneous emphysema (dashed arrow). (D) Thoracic MDCT axial slide shows an innominate artery traumatic dissection (solid arrow) with mild anterior mediastinal haematoma (asterisk).

The most common mechanism of blunt trauma was motor-vehicle crash (8 patients, $47 \%$ ) followed by motorcycle crash (7 patients, $41 \%$ ). There were not aortic injuries at the ascending aorta or the aortic arch due to falls or pedestrian-vehicle accidents in our series.

Trauma patients sustaining aortic injuries at the ascending aorta or the aortic arch presented a high proportion of critical (AIS $\geq 5$ ) thoracic injuries (11 patients, 65\%). In fact, these patients commonly associated myocardial contusion (7 patients, 41\%), haemopericardium (6 patients, 35\%) and sternal fracture (3 patients, $18 \%)$. In this study only 2 patients $(12 \%)$ with injury of the ascending aorta developed a significant (greater than moderate) AR.

A large number of major coexisting thoracic and extrathoracic injuries were recorded. All these trauma patients with injuries at the ascending aorta or the aortic arch had at least one severe extrathoracic injury with AIS $>3$. Severe head and neck injuries (AIS >3) were present in 11 patients $(65 \%)$. The percentage of patients with spinal cord injury was also high (4 patients, 23\%). The proportion of associated intra-abdominal injuries was so high as $47.1 \%$ (8 patients).

Trauma patients sustaining aortic injuries at the ascending aorta or the aortic arch presented a severely compromised haemodynamic status on admission regarding arterial hypotension (12 patients, $71 \%$ ) and need of endotracheal intubation at the site of accident or during transportation (12 patients, $71 \%$ ). A proportion of $41 \%$ ( 7 patients) had a GCS $<9$ on admission.

The type of aortic injury (I-IV) is shown on Table 2. 
Table 2. Classification of traumatic aortic injuries according to their severity in type I (intimal tear), type II (intramural haematoma), type III (pseudoaneurysm), or type IV (rupture). ${ }^{11}$

\begin{tabular}{ll}
\hline Type of aortic injury & Patients $(n, \%)(N=17)$ \\
\hline Type I & $3(17)$ \\
Type II & $4(24)$ \\
Type III & $6(35)$ \\
Type IV & $4(24)$ \\
\hline
\end{tabular}

Twelve patients $(71 \%)$ with traumatic injury of the ascending aorta or the aortic arch received a conservative management because of low-risk aortic injury (intimal tear $<10 \mathrm{~mm}$ or intramural haematoma without intimal tear; types I and II injuries) in 7 cases, and in the remaining 5 patients because of severe associated injuries, advanced age, or other severe premorbid conditions. Two of the aforementioned non-operatively managed patients were the only patients who presented a significant AR in the study, but these patients did not undergo a surgical repair because of associated critical brain injuries. Patients conservatively managed suffered a higher proportion of severe head and spinal cord injuries and presented more frequently haemodynamic instability than patients who underwent a surgical aortic repair, although those differences did not reach statistical significance (Table 3). Moreover, inhospital expected mortality and prognosis were worse in the subgroup of non-operative management as reflected by ISS, RTS and TRISS scores on admission. On the contrary, the proportion of high-degree life-threatening aortic injuries was higher in the subgroup of surgical management.

Table 3. Comparison of epidemiological and clinical characteristics between patients conservatively managed and patients who underwent a surgical aortic repair. The $p$ value of proportions analysis was obtained with Fisher's exact test, while $p$ value mean analysis corresponds to Student's $t$-test. (ISS = Injury Severity Score (ISS); RTS = Revised Trauma Score; TRISS = Trauma Injury Severity Score).

\begin{tabular}{|c|c|c|c|}
\hline Age (years) (mean (SD)) & $51(18.5)$ & $42.6(21.2)$ & 0.39 \\
\hline ISS (mean (SD)) & $53.2(22.9)$ & $36.4(13.1)$ & 0.038 \\
\hline RTS (mean (SD)) & $5(1.7)$ & $6.5(2)$ & 0.92 \\
\hline TRISS (mean (SD)) & $70.8(32.9)$ & $35.7(34.4)$ & 0.53 \\
\hline Arterial hypotension & $9(75 \%)$ & $3(60 \%)$ & 0.94 \\
\hline Need of endotracheal intubation & $9(75 \%)$ & $3(60 \%)$ & 0.94 \\
\hline Low-degree aortic injury (types I and II) & $6(50 \%)$ & $1(20 \%)$ & 0.56 \\
\hline $\begin{array}{l}\text { High-degree aortic injury (types III and } \\
\text { IV) }\end{array}$ & $6(50 \%)$ & $4(80 \%)$ & 0.56 \\
\hline In-hospital mortality & $8(67 \%)$ & $1(20 \%)$ & 0.12 \\
\hline
\end{tabular}

Among the 12 patients with conservative management, in-hospital mortality occurred in 3 patients with high-risk ATAI (types III and IV) because of aortic-related complications (cardiac tamponade in 2 cases and hypovolemic shock in 1 case), and 5 patients because of extrathoracic injuries (hypovolemic shock in 2 cases from an intraabdominal bleeding; brain herniation in 2 cases and multisystem organ failure in 1 case).

Five patients ( 2 ascending aorta and 3 aortic arch injuries) underwent an open repair through a median sternotomy ( 4 cases) and a thoracosternotomy ( 1 case). All cases were performed under CPB which was established by arterial cannulation of common femoral artery in 3 cases and right axillary artery in 2 patients. Patients with femoral artery cannulation required only a short ascending aorta replacement with a Dacron graft allowing distal aortic cross-clamping. In contrast, the 2 patients with axillary cannulation required circulatory arrest and ACP. One case presented a distal ascending aorta injury entailing a 
hemiarch replacement, whereas the other case suffered a complete aortic arch transection just proximal to the left common carotid origin with a large haematoma surrounding the arch and extending proximally to the distal ascending aorta and distally to the origin of the left subclavian artery. In the latter, the whole aortic arch was replaced under DHCA from distal ascending aorta to proximal descending aorta with a prosthetic Dacron graft with 4 branches (Intergard ${ }^{\circledR}$, Maquet GmbH \& Co., Germany). Three of the branches were used to reconstruct the arch branch vessels.

Among these 5 patients who were operated on, there was 1 postoperative death $(20 \%)$ due to a multisystem organ failure. None of the surgically managed patients presented a significant AR which required repair.

On the basis of the time from injury to definitive aortic repair, an emergent open surgical repair was required in $29 \%$ of patients ( 5 cases).

Expected mortality on admission was $\geq 50 \%$ according to an ISS $\geq 50$ points in 8 patients $(47.1 \%)$, while overall mean expected death rate calculated by TRISS was 59.7 (SD 38.6). Nevertheless, observed in-hospital mortality was 53\% (9 patients). Causes of in-hospital mortality in were: hypovolemic shock in 3 cases; cardiac tamponade in 2 cases; multisystem organ failure in 2 cases, and brain herniation in 2 cases.

After hospital discharge, clinical and imaging follow-up was available in all patients at a mean time of 25.4 (SD 36.7) months (range 0-120 months). Long-term survival was $46 \%$ at one year, $39 \%$ at five years, and $19 \%$ at ten years (Fig. 3). There were two late deaths after hospital discharge due to non-aortic related causes. One patient died of a lung cancer 36 months after hospital discharge, and the other, a patient with major cardiovascular risk factors and known 3 vessels coronary artery disease, died of a myocardial infarction 77 months after the trauma.

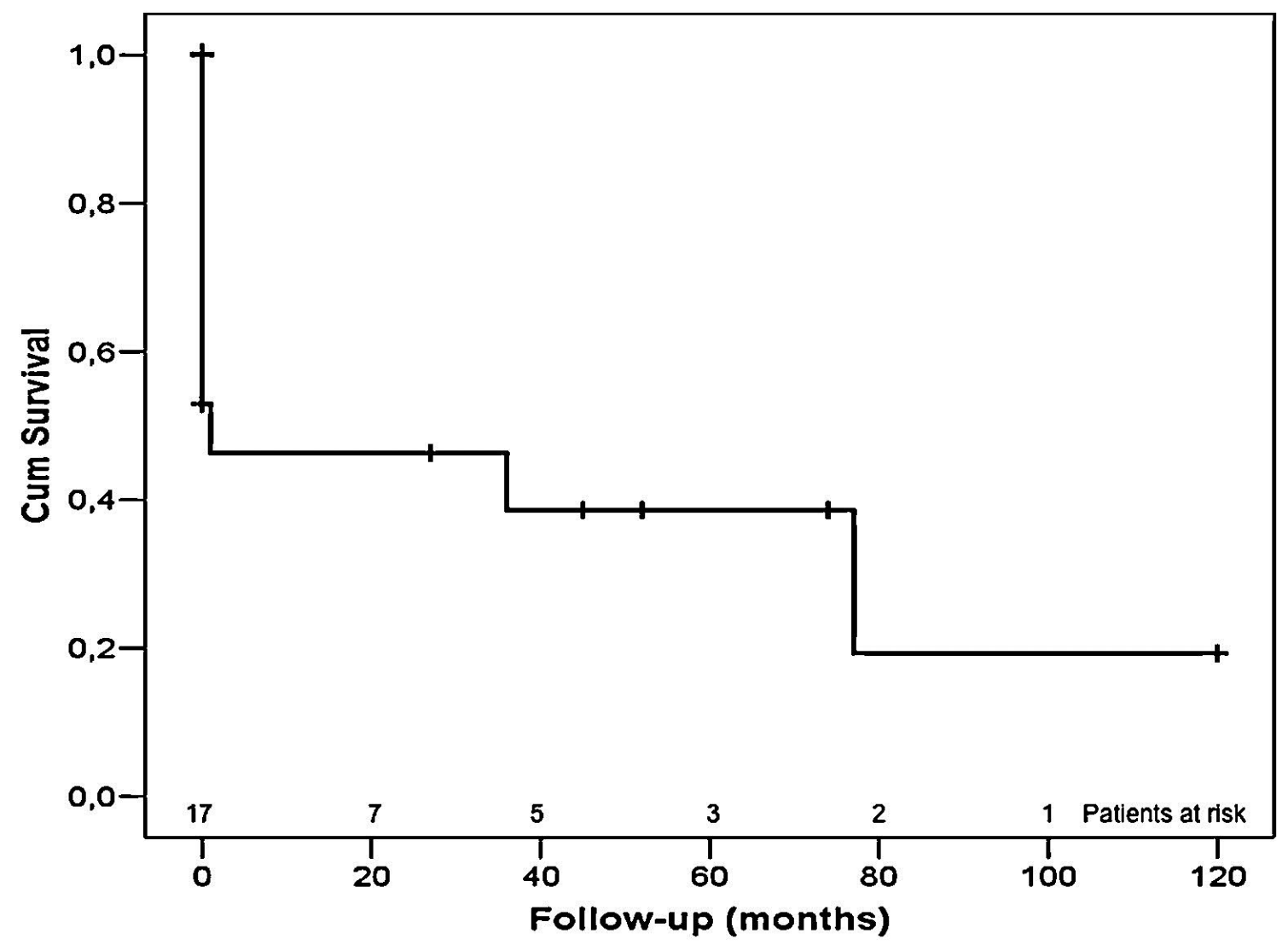

Fig. 3. Kaplan-Meier survival curves of patients with traumatic aortic injury of the ascending aorta or the aortic arch, including inhospital mortality. 


\section{Discussion}

The present study delineates several epidemiological features of blunt traumatic injuries of the ascending aorta and the aortic arch. This study also demonstrates that major trauma patients sustaining ATAIs at the ascending aorta or the arch present a characteristic profile regarding the prognosis, management and number and distribution of associated injuries.

Traumatic injuries of the ascending aorta and the aortic arch are more common in penetrating trauma, whereas injuries of the isthmus and descending aorta are more frequent in blunt trauma. ${ }^{4}$ In our series, ATAIs of the ascending aorta or the arch represented $20 \%$ of the overall number of trauma patients with ATAI admitted to the participating institutions during the period 2000-2010 (4 ascending aorta (5\%) and 13 aortic arch (15.3\%) injuries). It is a slightly higher proportion of injuries of the ascending aorta and arch than the ones reported in other clinical studies. The locations of the aortic injuries were similar in the two multicentre studies sponsored by the American Association for the Surgery of Trauma (AAST) and involved the ascending thoracic aorta/aortic arch in $7 \%$ in the $\mathrm{AAST}_{1}$ study ${ }^{15}$ and $5.4 \%$ in the $\mathrm{AAST}_{2}$ study. ${ }^{16}$ and 17 Cardarelli et al. published their experience managing ATAI and reported only 2 ascending aorta and no arch injuries among 219 blunt trauma patients with ATAIs. ${ }^{18}$

In 1990, Eddy et al., ${ }^{19}$ reviewed a series of 104 patients admitted to Harborview Medical Centre with ATAI over a 15-year period (1975-1990) reporting no ascending aorta traumatic injury and 7 patients (7\%) with arch injuries. In 2006, Cook et al. published the same institution series from 2000 to 2005, presenting only one injury of the ascending aorta (1.8\%) and 3 patients $(5.7 \%)$ with arch injuries among 54 patients with ATAIs. ${ }^{1}$ The authors pointed out that the advances in restraints might be responsible for the reduction in the incidence of ascending aorta and arch traumatic injuries when comparing the 2 time periods. ${ }^{1}$

Aortic arch injuries are remarkably more frequent in autopsy studies than in clinical practice. In a review of 217 blunt trauma autopsies published by Dosios et al., the analysis of time of death with regard to the anatomic location of ATAI revealed that a greater percentage of victims with injuries at the isthmus or descending thoracic aorta reached the hospital alive in comparison with those injured at the ascending aorta or the arch. ${ }^{4}$ That series reported 57 ascending aorta (21.6\%) and 18 aortic arch (8.3\%) traumatic injuries. ${ }^{4}$ More recently, Teixeira et al. reported a series of 104 blunt trauma fatal victims with associated ATAI, of which $3 \%$ were located at the ascending aorta and $11 \%$ at the arch. ${ }^{5}$

After demonstrating that the incidence of injury at the aortic isthmus in autopsy studies was lower than that demonstrated in clinical studies, several authors have suggested that non-isthmus injuries of the aorta are more lethal. ${ }^{4,5,20 \text { and } 21}$ Clinical and autopsy series seem to concur with the poor prognosis of traumatic injuries of the ascending aorta and aortic arch. Eddy et al. reported an in-hospital mortality of traumatic injuries of the ascending aorta or the arch as high as $81 \%$ between 1975 and $1990,{ }^{19}$ while it decreased to a $33 \%$ in the series published by Cook et al. between 2000 and 2005. ${ }^{1}$ In our series, inhospital mortality for ascending aorta or aortic arch injuries was over 50\%. Indeed, patients with ascending aorta or arch injuries presented a remarkably high-expected mortality on admission, as reflected by both TRISS and ISS scores on admission. The grave immediate prognosis of injuries located at ascending aorta and arch is probably the result of the frequent lethal concomitant intrathoracic injuries. In our series, associated thoracic trauma included myocardial contusion and haemopericardium in $41 \%$ and 35\% of ascending aorta and arch cases, respectively. Furthermore, a significant AR was developed in $12 \%$ of patients with injuries at the ascending aorta as a consequence of the thoracic trauma.

We also found that trauma patients with a traumatic injury at the ascending aorta or the aortic arch sustained a significant number of extrathoracic injuries. Severe head and neck injuries (AIS >3) and spinal cord injuries were present in $65 \%$ of the patients. On the other hand, the cause of death was directly related to the ATAI in 4 cases (45\%), head and abdominal injuries being the cause of death in the remaining 5 cases (55\%). This emphasises the fact that associated injuries can be just as lethal as the aortic injury; thus, treatment priorities should be modulated on an individual basis.

In the last decade, there have been several substantial diagnostic and therapeutic advances changing the management of ATAIs. Nowadays, open surgery still remains as the gold standard for treating traumatic ascending aorta and arch injuries. Definitive management of traumatic injuries of the ascending aorta or the aortic arch usually involves an open surgical repair (5 cases in our series, 29\%), which associates a not negligible surgical mortality (1 case in our series, 20\%). ${ }^{19}, 22$ and 23 In our series, we advocated for a surgical repair only in patients with a life-threatening or high degree aortic injury who also did not present a bad prognosis neurological injury. Only one patient with a low-degree aortic injury (intramural haematoma, type II) underwent a surgical repair due to the concomitant presence of pericardial effusion. Patients with severe head injuries were usually rejected for a surgical approach even in the presence of a high-degree aortic injury. Only one patient with a severe head injury was operated on and eventually died in the postoperative course. 
We chose femoral artery cannulation (3 patients) for saving time for institution of CPB in critically unstable patients. Nevertheless, when we expect the need of circulatory arrest, we have advocated for right axillary artery cannulation which simultaneously allows CPB establishment and ACP during the circulatory arrest (2 patients). The advent of TEVAR and the shift towards the delayed surgical treatment after stabilisation of other associated critical injuries have enabled a revolution in the management of ATAI in major trauma patients leading to a low in-hospital mortality in most current series. In fact, TEVAR has found itself a great role in descending aortic injuries. ${ }^{24}$ However, neither in this series nor in other published series in the literature has the endovascular treatment improved the prognosis of traumatic injuries of the ascending aorta or the arch. ${ }^{15}$ and 25 Although the contribution of TEVAR to the treatment of traumatic ascending aorta and aortic arch injuries was minor, improved trauma care, pre-hospital resuscitation, the widespread use of CT as a screening tool ${ }^{26}$ and clinical management with aggressive blood pressure control ${ }^{25,27}$ and 28 have altered the mortality rate of these injuries in the last years as reported by several authors. ${ }^{1,3}$ and 16 Nevertheless, the spread of hybrid surgery, which is gaining use by surgeons treating complex non-traumatic aortic arch pathologies, ${ }^{29}$ and 30 may allow surgeons to treat traumatic arch injuries that would otherwise be inoperable and to reduce the operative mortality of such complex traumatic injuries.

In our series, 12 patients $(71 \%)$ received a non-operative management. Basically, indications for a conservative approach are low-risk aortic injuries (intimal tear or intramural haematoma without tear), ${ }^{11}$ or high-risk patients with severe associated non-aortic traumatic injuries, comorbidities or advanced age. Patients conservatively managed presented a higher proportion of neurological injuries and associated a worse prognosis according to their extrathoracic injuries. As we have previously reported, ${ }^{25}$ the natural history of traumatic aortic injuries conservatively managed reveals a marked trend to develop late aorticrelated complications and the potential for rapid progression of the injuries mandates serial radiological controls during the first three months after injury and diagnosis and then annually whenever a conservative approach is selected. In this multicentre series, among the 12 patients with ascending aorta/arch injury and conservative management, in-hospital mortality occurred in 3 patients with high-risk ATAI (types III and IV) because of aortic-related complications, and 5 patients because of extrathoracic injuries. In spite of a low long-term survival in patients with ascending aorta/arch injuries, none of surviving patients with low-risk injuries experienced any aortic-related complications thanks to a strict control of blood pressure and cardiac contractility and a close imaging surveillance.

\section{Limitations}

This study presents the limitations inherent to any retrospective series. Although, in our opinion, the patient population in the present study reflects the wide clinical spectrum of ATAI at the ascending aorta and the aortic arch seen in major trauma patients and, also considering that ATAIs at those anatomic locations are rare in clinical series, the total number of patients is generally small and the present study may lack sufficient statistical power to determine with confidence some clinical relevant differences. The survival data beyond 1 year for patients with ascending aorta/arch injuries may not be meaningful, inasmuch as there were only 3 patients at risk at 5 years and only 1 at risk at 10 years. However, the strength of this paper lies in some new insights about the clinical characteristics and outcomes of traumatic aortic injuries of the ascending aorta and the aortic arch.

\section{Conclusions}

Traumatic injuries of the ascending aorta or the aortic arch present a high incidence of both intrathoracic and neurological injuries. Beyond the high aortic-related in-hospital mortality of the ascending aorta and arch injuries, these patients' associated injuries can be just as lethal as the aortic injury, and treatment priorities should be modulated on an individual basis. The suitability of an open surgical repair of traumatic injuries of the ascending aorta or the aortic arch injuries should be established according to their extrathoracic injuries which usually determine the patient's vital prognosis. The advances in trauma care and pre-hospital resuscitation, as well as in diagnostic tests, have improved the poor prognosis of these patients. Although the advent of TEVAR has had a negligible impact in the treatment of ascending aorta and arch traumatic injuries compared to the revolution experienced in the treatment of injuries at the aortic isthmus and descending aorta, the spread of aortic hybrid surgery may pose an attractive alternative treatment for complex arch injuries. 


\section{Conflicts of interest}

The authors state that there are no potential conflicts of interest to be considered in this article.

\section{References}

1. J. Cook, C. Salerno, B. Krishnadasan, S. Nicholls, M. Meissner, R. Karmy-Jones. The effect of changing presentation and management on the outcome of blunt rupture of the thoracic aorta. J Thorac Cardiovasc Surg, 131 (March (3)) (2006), pp. 594-600.

2. S.E. Mirvis, K. Shanmuganathan. Diagnosis of blunt traumatic aortic injury 2007: still a nemesis. Eur J Radiol, 64 (October (1)) (2007), pp. 27-40.

3. D. Demetriades, G.C. Velmahos, T.M. Scalea, G.J. Jurkovich, R. Karmy-Jones, P.G. Teixeira, et al. Diagnosis and treatment of blunt thoracic aortic injuries: changing perspectives. J Trauma, 64 (6) (2008), pp. 1415-1418 [discussion 1418-9].

4. T.J. Dosios, N. Salemis, D. Angouras, E. Nonas. Blunt and penetrating trauma of the thoracic aorta and aortic arch branches: an autopsy study. J Trauma, 49 (4) (2000), pp. 696-703.

5. P.G. Teixeira, K. Inaba, G. Barmparas, C. Georgiou, C. Toms, T.T. Noguchi, et al. Blunt thoracic aortic injuries: an autopsy study. J Trauma, 70 (January (1)) (2011), pp. 197-202.

6. K.L. Mattox, M.J. Wall Jr.. Historical review of blunt injury to the thoracic aorta. Chest Surg Clin N Am, 10 (1) (2000), pp. 167-182.

7. S.P. Baker, B. O’Neill, W. Haddon Jr., W.B. Long. The injury severity score: a method for describing patients with multiple injuries and evaluating emergency care. J Trauma, 14 (3) (1974), pp. 187-196.

8. H.R. Champion, W.J. Sacco, W.S. Copes, D.S. Gann, T.A. Gennarelli, M.E. Flanagan. A revision of the Trauma Score. J Trauma, 29 (5) (1989), pp. 623-629

9. H.R. Champion, W.J. Sacco, W.S. Copes. Injury severity scoring again. J Trauma, 38 (1) (1995), pp. 94-95.

10. V.X. Mosquera, M. Marini, J. Muñiz, V. Asorey-Veiga, B. Adrio-Nazar, R. Boix, et al. Traumatic aortic injury score (TRAINS): an easy and simple score for early detection of traumatic aortic injuries in major trauma patients with associated blunt chest trauma. Intensive Care Med (May) (2012).

11. W.A. Lee, J.S. Matsumura, R.S. Mitchell, M.A. Farber, R.K. Greenberg, A. Azizzadeh, et al. Endovascular repair of traumatic thoracic aortic injury: clinical practice guidelines of the Society for Vascular Surgery. J Vasc Surg, 53 (January (1)) (2011), pp. 187-192.

12. R.O. Bonow, B.A. Carabello, K. Chatterjee, A.C. de Leon Jr., D.P. Faxon, M.D. Freed, et al. 2008 focused update incorporated into the ACC/AHA 2006 guidelines for the management of patients with valvular heart disease: a report of the American College of Cardiology/American Heart Association Task Force on Practice Guidelines (Writing Committee to revise the 1998 guidelines for the management of patients with valvular heart disease). Endorsed by the Society of Cardiovascular Anesthesiologists, Society for Cardiovascular Angiography and Interventions, and Society of Thoracic Surgeons. J Am Coll Cardiol, 52 (September (13)) (2008), pp. e1-e142.

13. S.A. Glantz. It is all in the numbers. J Am Coll Cardiol, 21 (March (3)) (1993), pp. 835-837.

14. E. von Elm, D.G. Altman, M. Egger, S.J. Pocock, P.C. Gotzsche, J.P. Vandenbroucke. Strengthening the Reporting of Observational Studies in Epidemiology (STROBE) statement: guidelines for reporting observational studies. BMJ, 335 (October (7624)) (2007), pp. 806-808.

15. T.C. Fabian, J.D. Richardson, M.A. Croce, J.S. Smith Jr., G. Rodman Jr., P.A. Kearney, et al. Prospective study of blunt aortic injury: Multicenter Trial of the American Association for the Surgery of Trauma. J Trauma, 42 (3) (1997), pp. 374-380 [discussion 380-3].

16. D. Demetriades, G.C. Velmahos, T.M. Scalea, G.J. Jurkovich, R. Karmy-Jones, P.G. Teixeira, et al. Blunt traumatic thoracic aortic injuries: early or delayed repair - results of an American Association for the Surgery of Trauma prospective study. J Trauma, 66 (4) (2009), pp. 967-973.

17. D. Demetriades, G.C. Velmahos, T.M. Scalea, G.J. Jurkovich, R. Karmy-Jones, P.G. Teixeira, et al. Operative repair or endovascular stent graft in blunt traumatic thoracic aortic injuries: results of an American Association for the Surgery of Trauma Multicenter Study. J Trauma, 64 (3) (2008), pp. 561-570 [discussion 570-1].

18. M.G. Cardarelli, J.S. McLaughlin, S.W. Downing, J.M. Brown, S. Attar, B.P. Griffith. Management of traumatic aortic rupture: a 30-year experience. Ann Surg, 236 (4) (2002), pp. 465-469 [discussion 469-70].

19. A.C. Eddy, V.W. Rusch, T. Marchioro, D. Ashbaugh, E.D. Verrier, D. Dillard. Treatment of traumatic rupture of the thoracic aorta. A 15-year experience. Arch Surg, 125 (10) (1990), pp. 1351-1355 [discussion 1355-6].

20. J.D. Feczko, L. Lynch, J.E. Pless, M.A. Clark, J. McClain, D.A. Hawley. An autopsy case review of 142 nonpenetrating (blunt) injuries of the aorta. J Trauma, 33 (6) (1992), pp. 846-849.

21. H.M. Burkhart, G.A. Gomez, L.E. Jacobson, J.E. Pless, T.A. Broadie. Fatal blunt aortic injuries: a review of 242 autopsy cases. J Trauma, 50 (1) (2001), pp. 113-115.

22. P.J. Symbas, W.S. Horsley, P.N. Symbas. Rupture of the ascending aorta caused by blunt trauma. Ann Thorac Surg, 66 (July (1)) (1998), pp. 113-117.

23. Y.M. Carter, R.C. Karmy-Jones, D.C. Oxorn, G.S. Aldea. Traumatic disruption of the aortic arch. Eur J Cardiothorac Surg, 20 (December (6)) (2001), p. 1231

24. E.S. Xenos, N.N. Abedi, D.L. Davenport, D.J. Minion, O. Hamdallah, E.E. Sorial, et al. Meta-analysis of endovascular vs open repair for traumatic descending thoracic aortic rupture. J Vasc Surg, 48 (2008), pp. 13431351. 
25. V.X. Mosquera, M. Marini, J.M. Lopez-Perez, J. Muniz-Garcia, J.M. Herrera, I. Cao, et al. Role of conservative management in traumatic aortic injury: comparison of long-term results of conservative, surgical, and endovascular treatment. J Thorac Cardiovasc Surg, 142 (September (3)) (2011), pp. 614-621.

26. S.D. Steenburg, J.G. Ravenel, J.S. Ikonomidis, C. Schonholz, S. Reeves. Acute traumatic aortic injury: imaging evaluation and management. Radiology, 248 (September (3)) (2008), pp. 748-762.

27. T.C. Fabian, K.A. Davis, M.L. Gavant, M.A. Croce, S.M. Melton, J.H. Patton Jr., et al.. Prospective study of blunt aortic injury: helical CT is diagnostic and antihypertensive therapy reduces rupture. Ann Surg, 227 (5) (1998), pp. 666-676 [discussion 676-7].

28. J.W. Pate, M.L. Gavant, D.S. Weiman, T.C. Fabian. Traumatic rupture of the aortic isthmus: program of selective management. World J Surg, 23 (1) (1999), pp. 59-63.

29. R. Gottardi, M. Funovics, N. Eggers, A. Hirner, M. Dorfmeister, J. Holfeld, et al. Supra-aortic transposition for combined vascular and endovascular repair of aortic arch pathology. Ann Thorac Surg, 86 (November (5)) (2008), pp. 1524-1529.

30. M. Schoder, J. Lammer, M. Czerny. Endovascular aortic arch repair: hopes and certainties. Eur J Vasc Endovasc Surg, 38 (September (3)) (2009), pp. 255-261. 